Supporting Information for

\title{
Uncovering the Charge Transfer between Carbon Dots and Water by in situ soft X-ray Absorption
}

\section{Spectroscopy}

Jian Ren, ${ }^{1,2}$ Demetra S. Achilleos, ${ }^{3}$ Ronny Golnak, ${ }^{4}$ Hayato Yuzawa, ${ }^{5}$ Jie Xiao, ${ }^{4}$ Masanari Nagasaka, ${ }^{5}$ Erwin Reisner, ${ }^{3}$ Tristan Petit ${ }^{1 *}$

${ }^{1}$ Institute for Nanospectroscopy, Helmholtz-Zentrum Berlin für Materialien und Energie GmbH (HZB), Albert-Einstein-Straße 15, 12489 Berlin, Germany

${ }^{2}$ Department of Physics, Freie Universität Berlin, Arnimallee 14, 14195 Berlin, Germany

${ }^{3}$ Christian Doppler Laboratory for Sustainable SynGas Chemistry, Department of Chemistry, University of Cambridge, Lensfield Road, Cambridge CB2 1EW, UK

${ }^{4}$ Department of Highly Sensitive X-ray Spectroscopy, Helmholtz-Zentrum Berlin für Materialien und Energie GmbH (HZB), Albert-Einstein-Straße 15, 12489 Berlin, Germany

${ }^{5}$ Institute for Molecular Science, Myodaiji, Okazaki 444-8585, Japan

*E-mail: tristan.petit@helmholtz-berlin.de

\section{Experimental Section}

Table S1. Fitting details of the C K edge TEY-XA spectra of CDs samples.

Figure S1. Deconvolution of the C K-edge TEY-XA spectra of CDs samples.

Figure S2. O K-edge TIY-XA spectra of $a$-CD, $g-\mathrm{N}-\mathrm{CD}$ and water (top) and O K-edge TEY-XA spectra of the three CDs samples (bottom).

Figure S3. N K-edge TEY-XA spectrum of $g-\mathrm{N}-\mathrm{CD}$.

Table S2. Fitting details of the O K-edge transmission mode XA spectra of CDs samples.

Figure S4. Deconvolution of the O K-edge transmission mode XA spectra of CDs samples. 


\section{Experimental Section}

Synthesis of carbon dots: The three CD samples were synthesized via the single-source precursor approach. Aspartic and citric acid were used as the precursors for the synthesis of the nitrogen doped and undoped CDs, respectively. The nanostructure of the CD core varies from amorphous to graphitic depending on the temperature of synthesis employed. More specifically, amorphous CDs were synthesized at $180{ }^{\circ} \mathrm{C}$ whereas the graphitic samples at $320^{\circ} \mathrm{C}$.

There is no further functionalization process after the thermolysis reaction, the functionalities of CDs samples are therefore inherited from the precursor molecules. The samples surfaces are decorated with a variety of carbonyl groups. The nitrogen incorporation occurs in the core of $g-\mathrm{N}-\mathrm{CD}$. The $\mathrm{C}: \mathrm{N}$ ratio was found to be $3.8: 1$, as characterized by elemental analysis. ${ }^{1}$

All CDs samples are spherical nanoparticles. Their particle sizes were characterized by high-resolution TEM. For $a-\mathrm{CD}, g-\mathrm{CD}$ and $g-\mathrm{N}-\mathrm{CD}$, the average size are respectively $6.8 \pm 2.3$ $\mathrm{nm}, 3.6 \pm 1.0 \mathrm{~nm}$ and $3.1 \pm 1.1 \mathrm{~nm}$. Full synthetic and characterization details have been reported previously. $^{1,2}$

TIY mode XAS measurements: Experiments were conducted at the U49-2 PGM1 undulator beamline of BESSY II synchrotron using the LiXEdrom endstation with an electrochemical cell described in reference ${ }^{3}$. Carbon dots aqueous dispersions $(6 \mathrm{wt} \%)$ were characterized in a flow cell with two electrodes connecting to an ammeter. Thin silicon nitride membrane $(75 \mathrm{~nm})$ coated with $\mathrm{Au}(15 \mathrm{~nm})$ and $\mathrm{Cr}(5 \mathrm{~nm})$ was used to isolate the liquid from the vacuum and served as the front electrode. The back electrode is a platinum rod of $1.6 \mathrm{~mm}$ in diameter. The distance between the front and back electrodes is about $1 \mathrm{~mm}$. With X-ray light, the background current below the C K-edge was about in the order of $100 \mathrm{nA}$, and the detected signal of CDs samples was approximately 2-3 times larger. Before each scan, the flow of CDs dispersions was stopped for at least 30 minutes to allow the detected signals to stabilize. The carbon contamination from 
the beamline was corrected by measuring a reference TIY-XA spectrum at the C K-edge with pure water.

Transmission mode XAS measurements: The data were collected at the BL3U undulator beamline of UVSOR-III Synchrotron with a transmission liquid cell previously described. ${ }^{4,5}$ The CDs dispersion sample was sandwiched between two $100 \mathrm{~nm}$ thick $\mathrm{Si}_{3} \mathrm{~N}_{4}$ membranes (NTT AT Co., Ltd.) with pressed Teflon spacers set between the window frames of the membranes and can be substituted by other samples in combination with a tubing pump system. The thickness of liquid layer was optimized in order to transmit soft X-rays with an appropriate absorbance. The energy resolutions of incident soft X-rays at the $\mathrm{C}$ and $\mathrm{O} \mathrm{K}$-edge are 0.14 and $0.4 \mathrm{eV}$, respectively. The XA spectra are based on the Beer-Lambert law, $\ln \left(\mathrm{I}_{0} / \mathrm{I}\right)$, where $\mathrm{I}_{0}$ and $\mathrm{I}$ are the detection currents passed through the cell without and with samples, respectively. The liquid flow is stopped during the XA measurement because the samples are not sensitive to radiation damage under our experimental conditions. The calibration of the photon energies at the $\mathrm{C}$ and $\mathrm{O}$ K-edges made use of thin polymer films (150 nm or $300 \mathrm{~nm})$ with additional photodiode detector in the vacuum chamber. ${ }^{5}$ The photon energy of the polymer film at the C K-edge is calibrated by the first peak $(287.96 \mathrm{eV})$ of methanol molecules, and that at the $\mathrm{O} \mathrm{K}$-edge is calibrated by the $\mathrm{O} 1 \mathrm{~s}$ $\pi^{*}$ peak $(530.80 \mathrm{eV})$ for free $\mathrm{O}_{2}$ molecules, respectively. ${ }^{6}$ For the result at $\mathrm{C} \mathrm{K}$-edge, the background signal of the membrane recorded with pure water was subtracted from the sample spectra.

TEY mode XAS measurement: Solid samples were characterized by XAS using total electron yield (TEY) mode on CDs drop-casted on a conductive Si substrate. The data were collected at the U49-2_PGM1 beamline of the BESSY II synchrotron radiation source using the LiXEdrom endstation. 
Table S1. Fitting details of the C K edge TEY-XA spectra of CDs samples.

\begin{tabular}{ccccccc}
\hline \multirow{2}{*}{ Peak } & \multicolumn{2}{c}{$\boldsymbol{a}-\mathbf{C D}$} & \multicolumn{2}{c}{$\boldsymbol{g}$-CD } & \multicolumn{2}{c}{$\boldsymbol{g}$-N-CD } \\
\cline { 2 - 7 } & $\begin{array}{c}\text { Energy } \\
{[\mathbf{e V}]^{-}}\end{array}$ & $\begin{array}{c}\text { FWHM } \\
{[\mathbf{e V}]^{\mathbf{a})}}\end{array}$ & $\begin{array}{c}\text { Energy } \\
{[\mathbf{e V}]}\end{array}$ & $\begin{array}{c}\text { FWHM } \\
{[\mathbf{e V}]}\end{array}$ & $\begin{array}{c}\text { Energy } \\
{[\mathbf{e V}]^{-}}\end{array}$ & $\begin{array}{c}\text { FWHM } \\
{[\mathbf{e V}]}\end{array}$ \\
\hline A & 285.24 & 0.87 & 285.14 & 1.02 & 285.28 & 0.98 \\
B & 286.55 & 0.96 & 286.48 & 1.33 & 286.43 & 0.79 \\
C & 287.78 & 1.33 & 287.20 & 1.33 & 287.25 & 1.00 \\
D & $/$ & $/$ & $/$ & $/$ & 288.10 & 1.00 \\
E & 288.69 & 0.72 & 288.38 & 1.37 & 288.50 & 1.00 \\
F & 289.80 & 1.00 & 289.70 & 1.33 & 289.78 & 1.32 \\
G & 290.61 & 1.46 & 290.60 & 1.14 & 290.57 & 1.45 \\
H & $/$ & $/$ & 291.57 & 1.77 & 1 & $/$ \\
I & 295.84 & 7.49 & 295.93 & 5.63 & 296.10 & 7.99 \\
J & 302.10 & 5.98 & 301.11 & 5.48 & 303.23 & 6.01 \\
\hline
\end{tabular}

a) Full Width at Half Maximum (FWHM) of Gaussian peaks.

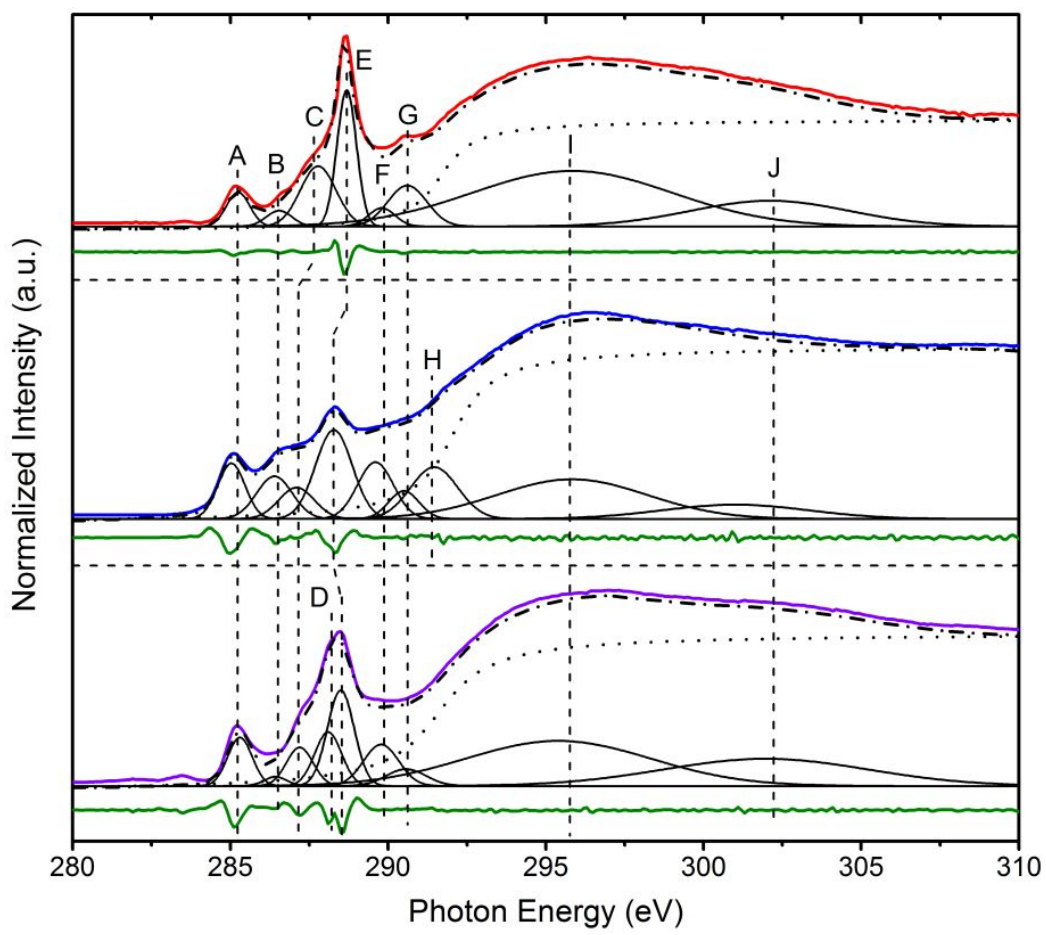

Figure S1. Deconvolution of the C K edge TEY-XA spectra of $a$-CD (red), $g$-CD (blue) and $g$-N-CD (violet) aqueous dispersions. The second derivatives (olive, dash dot) are also plotted. The different Gaussian peaks (solid), the resulting fit (dash dot) and the background (dot) are shown below the experimental spectra. The experimental spectra are offset for clarity. 


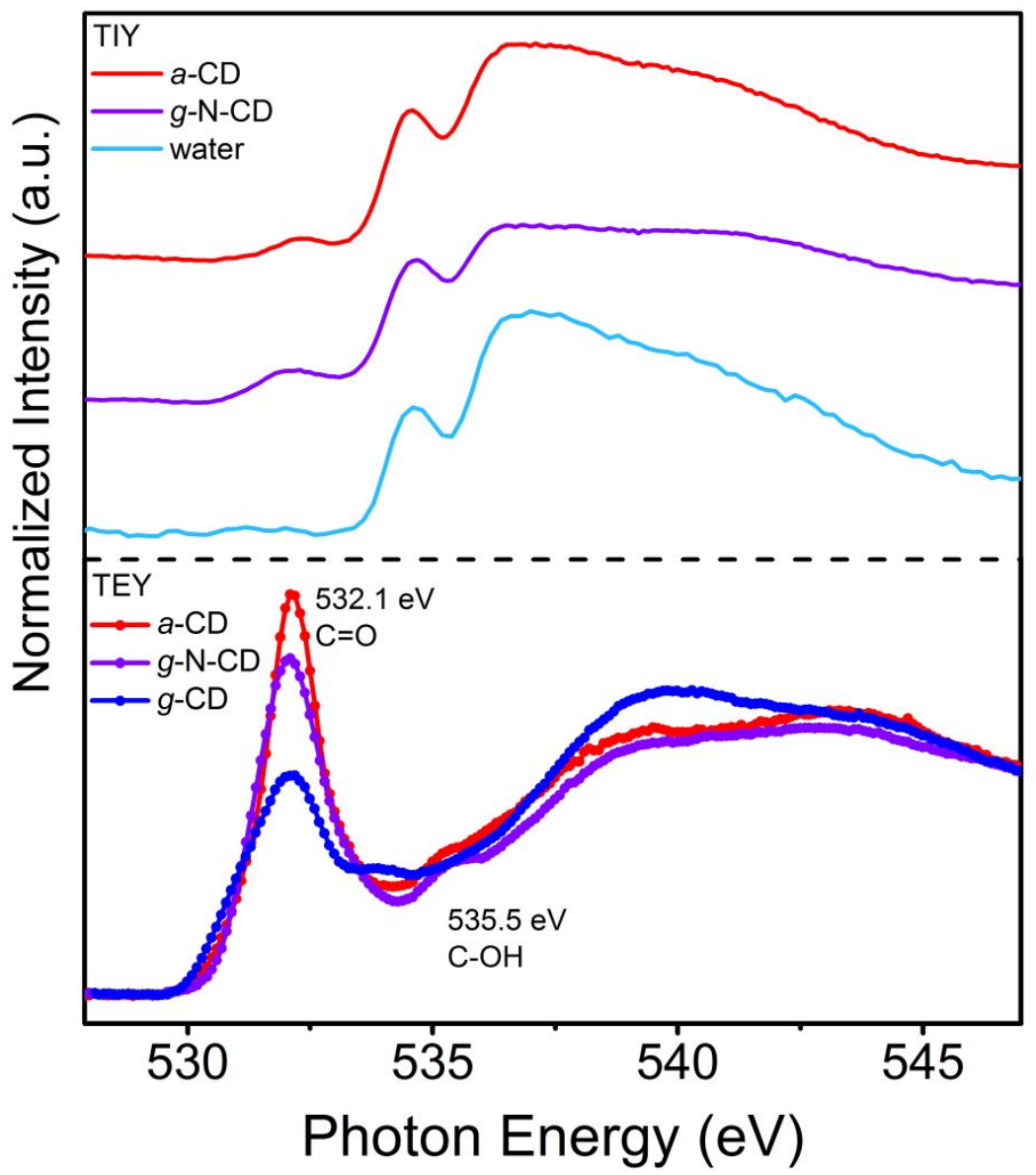

Figure S2. O K-edge TIY-XA spectra of $a-\mathrm{CD}, g-\mathrm{N}-\mathrm{CD}$ and water (top) and O K-edge TEY-XA spectra of the three CDs samples (bottom). 


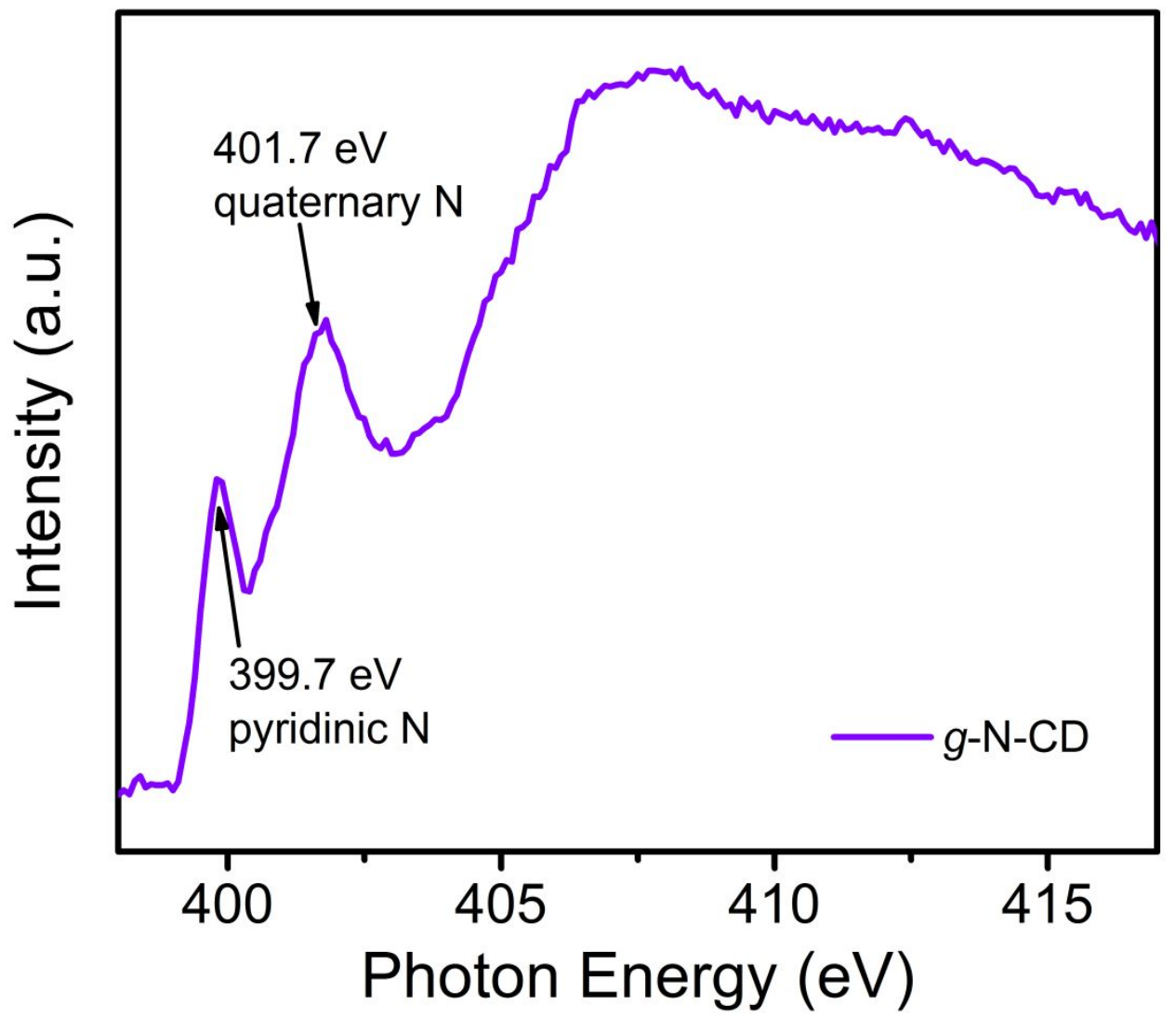

Figure S3. N K-edge TEY-XA spectrum of $g$-N-CD. 
Table S2. Fitting details of the O K edge transmission mode XA spectra of CDs samples.

\begin{tabular}{cccccccc}
\hline & & & \multicolumn{5}{c}{ Area } \\
\cline { 5 - 7 } Peak & $\begin{array}{c}\text { Energy } \\
{[\mathbf{e V}]}\end{array}$ & $\begin{array}{c}\text { FWHM } \\
{[\mathbf{e V}]}\end{array}$ & & $\boldsymbol{a}$-CD & $\boldsymbol{g}$-CD & $\boldsymbol{g}$-N-CD & water \\
\hline $\mathrm{A}$ & 532.16 & 1.14 & 0.0160 & $/$ & 0.0318 & $/$ \\
$\mathrm{B}$ & 534.51 & 0.87 & 0.0982 & 0.1524 & 0.2156 & 0.1366 \\
$\mathrm{C}$ & 536.98 & 3.28 & 0.7982 & 1.0030 & 1.5294 & 0.7007 \\
$\mathrm{D}$ & 540.49 & 4.95 & 1.1271 & 1.5256 & 2.2405 & 1.0723 \\
$\mathrm{E}$ & 544.70 & 2.58 & 0.0447 & 0.0467 & 0.0997 & $/$ \\
\hline
\end{tabular}

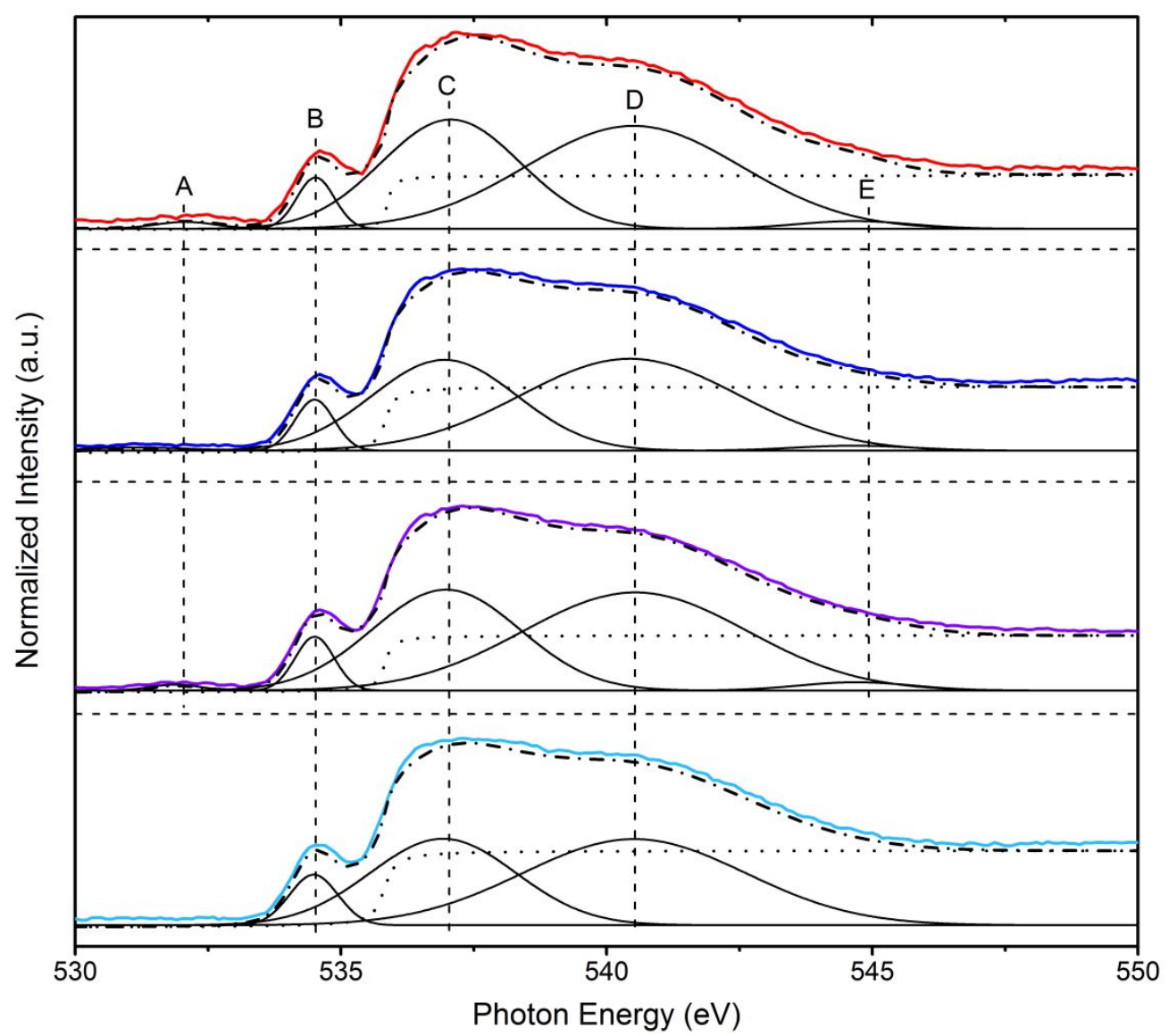

Figure S4. Deconvolution of the O K-edge transmission XA spectra of $a$-CD (red), $g$-CD (blue), $g$-N-CD (violet) aqueous dispersions and pure water (turquoise). The different Gaussian peaks (solid), the resulting fit (dash dot) and the background (dot) are shown below the experimental spectra. The experimental spectra are offset for clarity. 


\section{REFERENCES:}

(1) Martindale, B. C. M.; Hutton, G. A. M.; Caputo, C. A.; Prantl, S.; Godin, R.; Durrant, J. R.; Reisner, E. Enhancing Light Absorption and Charge Transfer Efficiency in Carbon Dots through Graphitization and Core Nitrogen Doping. Angew. Chemie 2017, 129, 65596563.

(2) Martindale, B. C. M.; Hutton, G. A. M.; Caputo, C. A.; Reisner, E. Solar Hydrogen Production Using Carbon Quantum Dots and a Molecular Nickel Catalyst. J. Am. Chem. Soc. 2015, 137, 6018-6025.

(3) Schön, D.; Xiao, J.; Golnak, R.; Tesch, M. F.; Winter, B.; Velasco-Velez, J.-J.; Aziz, E. F. Introducing Ionic-Current Detection for X-Ray Absorption Spectroscopy in Liquid Cells. $J$. Phys. Chem. Lett. 2017, 8, 2087-2092.

(4) Nagasaka, M.; Hatsui, T.; Horigome, T.; Hamamura, Y.; Kosugi, N. Development of a Liquid Flow Cell to Measure Soft X-Ray Absorption in Transmission Mode: A Test for Liquid Water. J. Electron Spectros. Relat. Phenomena 2010, 177, 130-134.

(5) Nagasaka, M.; Yuzawa, H.; Horigome, T.; Kosugi, N. Reliable Absorbance Measurement of Liquid Samples in Soft X-Ray Absorption Spectroscopy in Transmission Mode. $J$. Electron Spectros. Relat. Phenomena 2018, 224, 93-99.

(6) Nagasaka, M.; Mochizuki, K.; Leloup, V.; Kosugi, N. Local Structures of Methanol-Water Binary Solutions Studied by Soft X-Ray Absorption Spectroscopy. J. Phys. Chem. B 2014, $118,4388-4396$. 